\title{
Nachzustand, Distanz und Aspektualität als Komponenten einer formfokussierten Steuerung von Perfekt und Präteritum
}

\author{
Nicole Schumacher (Berlin)
}

\begin{abstract}
The fascinating complexity of the German past tenses has created many scientific contributions within the pedagogic, linguistic and SLA communities. Not often though the findings of the theoretical branches have been applied for pedagogic purposes. The article aims at bridging this gap by elaborating the semantic respectively pragmatic concepts of post-state, distance and aspect within a focus on form approach. The concepts of post-state and distance help to present systematically the different readings of the German present perfect and past tense. Reflections on aspectual meanings lead to insights into L2 decoding and coding preferences of learners whose L1 grammaticalises aspect. I propose several proactive and reactive focus on form activities (input flood, input enhancement, metalinguistic feedback, dictogloss) to increase the salience of the elaborated concepts within the L2 instruction of the German past tenses.
\end{abstract}

\section{Perfekt und Präteritum als Lerngegenstände in Deutsch als Fremdsprache}

Es herrscht eine bemerkenswerte Diskrepanz zwischen der Darstellung von Perfekt und Präteritum in Lehrmaterialien, der linguistischen Diskussion um diese beiden Tempora und den Schwerpunkten, die in Zweitspracherwerbsstudien zu Tempus gesetzt werden. Was macht diesen Tempusklassiker bloß so vielschichtig? Welche Dimensionen werden beschrieben und erforscht? Welche Forschungsergebnisse können - unter Berücksichtigung lerntheoretischer Erkenntnisse - didaktisch umgesetzt werden?

Ein zentraler Gegenstandsbereich in Lehrmaterialien und in der Linguistik besteht darin auszuloten, warum Perfekt und Präteritum in einigen Kontexten austauschbar sind, in anderen jedoch nicht. Hierfür wird in Lehrmaterialien nahezu ausschließlich die Dimension des Gebrauchs behandelt, vor allem werden stilistische und textsortenspezifische Phänomene angeführt. Die Formenbildung wird unabhängig hiervon behandelt. In einem großen Teil der linguistischen Diskussion hingegen steht die Frage im Zentrum, inwiefern semantische Faktoren einerseits und pragmatische Faktoren andererseits ausschlaggebend sind für Bedeutung und Gebrauch beider Tempora. Hierbei werden systematische Beschreibungen und Erklärungen vorgeschlagen, die Zusammenhänge von Formen und Bedeutungen beinhalten.

In meinen Augen ist es wichtig, auch für lern- und lehrtheoretische Zwecke zwischen semantischen und pragmatischen Dimensionen von Perfekt und Präteritum zu differenzieren sowie Zusammenhänge von Formen und Bedeutungen zu berücksichtigen. Denn Wissen über Gebrauchsbeschränkungen und Gebrauchspräferenzen gehört zu den Lernzielen im Fremdsprachenerwerb, und das Mapping von Formen und Bedeutungen ist zentraler Bestandteil von Lernprozessen beim Aufbau der L2-Kompetenz.

In der lern- und lehrtheoretischen Diskussion um spracherwerbsfördernde didaktische Strategien, die die traditionelle Dichotomie zwischen rein formbezogenen, strukturellen und rein 
bedeutungsbezogenen, kommunikativen Curricula überwunden hat, wird vielfach auf die verschiedenen Dimensionen einer formfokussierten Steuerung - formfocused instruction oder focus on form - hingewiesen (vgl. Doughty/Williams (eds.) 1998, Ellis (ed.) 2001, Fotos/Nassaji (ed.) 2007, Schoormann/Schlak 2010, Schifko 2011). Ausgangspunkt sind Beobachtungen, nach denen ein rein kommunikativer Unterricht mit ausreichendem Input und vielfältigen Interaktionsmöglichkeiten nicht ausreicht, damit Lernende eine native oder zielsprachennahe grammatische Kompetenz aufbauen. Wegen begrenzter Verarbeitungskapazitäten verarbeiten Lernende bei der Bewältigung kommunikativer Aufgaben primär die Bedeutung sprachlicher Ausdrücke. Um sie in ihrem Lernprozess auf dem Weg zu einer zielsprachennahen grammatischen Kompetenz zu unterstützen, ist es somit notwendig, auch die Formen sprachlicher Ausdrücke - im Zusammenhang mit ihren Bedeutungen - salient zu machen (vgl. Van Patten 2004, Wong 2005, Handwerker 2009, Handwerker/Madlener 2009: 31-37). Im vorliegenden Beitrag möchte ich Vorschläge dafür machen, welche FormBedeutungszusammenhänge von Perfekt und Präteritum in Lehrmaterialien und im Klassenzimmer salient gemacht werden sollten. Hier spielen die Bedeutungs- bzw. Gebrauchsdimensionen Nachzustand und Distanz eine zentrale Rolle.

Die Lesarten von Perfekt und Präteritum können neben temporalen auch aspektuale Bedeutungseffekte enthalten. Diese werden in Lehrmaterialien kaum behandelt. Wie die umfangreiche Literatur zu Tempus und Aspekt im Zweitspracherwerb zeigt, sind temporale und aspektuale jedoch Konzepte aufs Engste miteinander verbunden und prägen den Aufbau von temporalen Ausdrücken in Lernersprachen (vgl. Bardovi-Harlig 2000). In vielen Spracherwerbsstudien werden die Konzeptbereiche beleuchtet, die der Produktion und Rezeption von Tempora kognitiv zugrunde liegen. Hierbei spielen neben temporalen Konzepten wie Vorzeitigkeit, Gleichzeitigkeit und Nachzeitigkeit auch aspektuale Konzepte wie Abgeschlossenheit, Verlauf, Resultativität und Habitualität eine zentrale Rolle. Um eine spracherwerbsfördernde Steuerung zu gewährleisten, ist es auch für Lern- und Lehrzwecke sinnvoll, diese aspektuale Dimension zu berücksichtigen, denn diese ist häufig "durch die Grammatik [der L1] fokussiert" (vgl. von Stutterheim/Carroll 2007). Insbesondere durch in der jeweiligen L1 grammatikalisierte temporale und aspektuale Kategorien werden Lernende im Gebrauch von L2Ausdrücken beeinflusst. Das so geprägte Ausdrucksbedürfnis äußert eine englischsprachige Niederländischlernerin aus Starren (2001) sehr eindrücklich: "How can you live without a progressive?" (Starren 2001: 2).

Ziel meines Beitrags ist es, die drei Bedeutungs- bzw. Gebrauchsdimensionen Nachzustand, Distanz und Aspektualität herauszuarbeiten, die zentral für die Lerngegenstände Perfekt und Präteritum sind. In Abschnitt 2 zeige ich zunächst die Lesarten von Perfekt und Präteritum mit ihren Gebrauchsbeschränkungen und -präferenzen. Abschnitt 3 ist den Zusammenhängen von Formen und Bedeutungen gewidmet. Im vierten Abschnitt mache ich erste Vorschläge dafür, die zuvor beschriebenen Dimensionen als Komponenten einer formfokussierten Steuerung zu behandeln. Mein Beitrag schließt mit einem kurzen Ausblick auf weitere lern- und lehrtheoretische Forschungsfragen zur Formfokussierung bei den Tempora. 


\section{Gebrauchsbeschränkungen und Gebrauchspräferenzen: Die Lesarten}

Der zentrale semantisch-grammatische Unterschied zwischen Perfekt und Präteritum lässt sich daran erkennen, dass beide Tempora Vergangenheitslesarten zulassen, nur das Perfekt jedoch auch Resultatslesarten ermöglicht. Bei Vergangenheitslesarten wird eine Aussage über eine in der Vergangenheit liegende Situation gemacht, bei Resultatslesarten eine Aussage über den Nachzustand eines vorangegangenen Ereignisses, der in der Gegenwart oder der Zukunft liegt. ${ }^{1}$ Die folgenden beiden Abbildungen veranschaulichen die jeweils möglichen Lesarten.

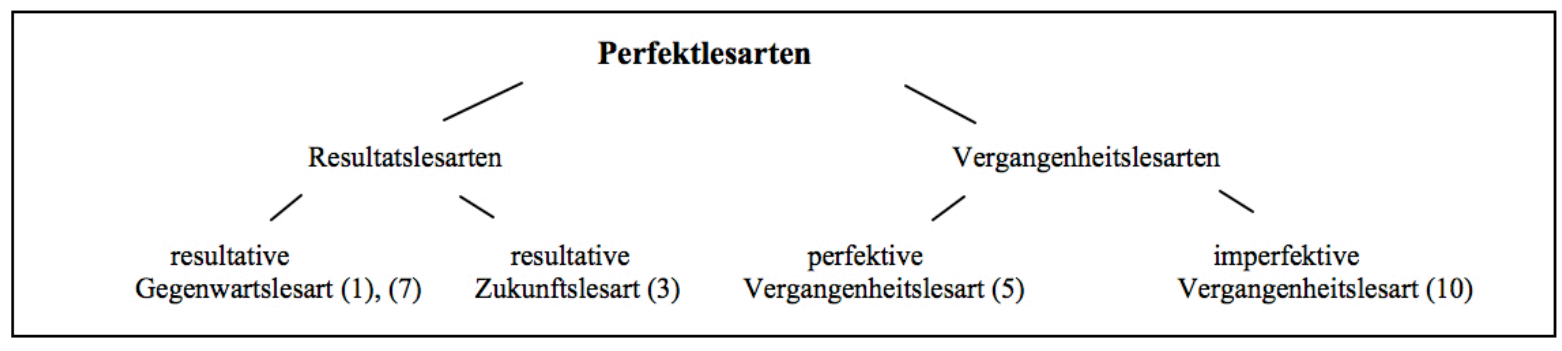

Abbildung 1: Perfektlesarten

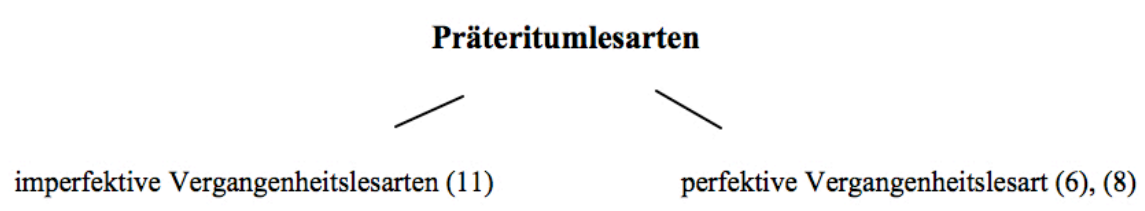

Abbildung 2: Präteritumlesarten

Einen ersten konkreten Hinweis auf Bedeutungsunterschiede zwischen Perfekt und Präteritum liefern die folgenden Beispiele. Sie zeigen, dass nicht jedes Perfekt durch ein Präteritum ersetzbar ist.

(1) Er hat sich damit jetzt als Politgangster entlarvt. (Hörbeleg Tagesschau 19.8.03)

(2) *Er entlarvte sich damit jetzt als Politgangster.

In (1) liegt eine resultative Gegenwartslesart des Perfekts vor: Es wird eine Aussage über den gegenwärtigen Nachzustand gemacht, in dem sich das Subjekt befindet. Dieser Zustand resultiert aus einer bestimmten Tätigkeit, die mit dem vorangehenden Ereignis des Entlarvens verbunden ist. Das telische Verb sich entlarven stellt lexikalisch diese beiden Phasen zur Verfügung: Der Tätigkeitsphase, die einen inhärenten Endpunkt enthält, folgt der Zustand des Subjektsreferenten, der seine wirklichen Absichten enthüllt hat. Resultatslesarten weisen oft telische Ausdrücke auf, weil sich in der Verbsemantik verankerte inhärente Endpunkte und ihre Nachzustände besonders gut dafür eignen, ins Zentrum der Aussage gerückt zu werden (vgl. Musan 2002: 97, Schumacher 2005: 164-168).

Wie wir an (2) sehen, ist ein solche Lesart mit dem Präteritum nicht möglich. Dies liegt daran, dass nur das Perfekt, nicht aber das Präteritum in seiner Konstruktionsbedeutung 2 die Komponente eines Nachzustands zur Verfügung stellt, die ins Zentrum einer Äußerung gerückt werden kann. Ein entsprechendes Phänomen ist am Vergleich zwischen (3) und (4) erkennbar.

(3) Gleich habe ich es geschafft.

\footnotetext{
${ }^{1}$ Vgl. für die theoretischen Grundlagen dieser Idee Musan $(1999,2002)$ sowie für ihre Anwendung in Deutsch als Fremdsprache Schumacher (2005).

${ }^{2}$ Dieser Begriff wird in Abschnitt 3 näher bestimmt.
} 
(4)

*Gleich schaffte ich es.

Auch in (3) wird eine Aussage über den Zustand des Subjekts getroffen. Dieser resultiert aus dem Ereignis des Schaffens und liegt in der Zukunft. Wiederum stellt das Verb schaffen lexikalisch einen Endpunkt zur Verfügung: den Punkt, an dem etwas zu Leistendes bewältigt ist und der entsprechende Nachzustand des Subjekts einsetzt. Eine solche resultative Zukunftslesart lässt ein Präteritum nicht zu, wie an (4) ersichtlich ist.

Bis hierher haben wir es mit Gebrauchsbeschränkungen zu tun, d.h mit den grammatischen und semantischen Unterschieden zwischen Perfekt und Präteritum. In (5) und (6) sehen wir hingegen Gebrauchspräferenzen.

(5) Genug ist genug. Er hat sich damit gestern endgültig als Politgangster entlarvt.

(6) Gestern passierte viel. Er entlarvte sich als Politgangster...

In beiden Fällen liegen Vergangenheitslesarten vor, und in grammatischer Hinsicht sind beide Tempora möglich. Es handelt sich um perfektive Vergangenheitslesarten. Die Situationen des Sich-Entlarvens werden als abgeschlossen, mit einer rechten Grenze, gleichsam "von außen" perspektiviert, um die gängigsten Metaphern für die Beschreibung des perfektiven Aspekts heranzuziehen (vgl. Klein 2009: 52-56; von Stutterheim/Carroll/Klein 2009: 198).

Die Differenz zwischen (5) und (6) ist nicht aspektual, sondern liegt in der subjektiven, sprecherbezogenen Dimension der Distanz begründet, die sich durch Weinrichs (1993) Konzepte des Erzählens und Besprechens erfassen lässt (vgl. auch Willkop 2003a, b). (6) ist eine typische schriftliche Erzählsituation, für die das Präteritum gewählt wird, um dem Leser eine entspannte Rezeptionshaltung nahezulegen: Es wird darum gebeten, eine - virtuelle - Diskussion über die einzelnen Äußerungen zunächst einmal aufzuschieben, um eine Erzählung entfalten zu können. In (5) hingegen, einer mündlich und schriftlich denkbaren Äußerung, wird das Perfekt gewählt, weil der Sprecher der vergangenen Situation Relevanz für die unmittelbare Gegenwart der Sprechsituation beimisst bzw. eine gespannte Rezeptionshaltung beim Leser evoziert und damit seine Bereitschaft signalisiert, über seine Äußerungen - virtuell - zu diskutieren (vgl. Weinrich 1993: 198-207). Der Gebrauchsunterschied zwischen beiden Tempora lässt sich darauf zurückführen, dass das Präteritum, nicht jedoch das Perfekt in seiner Konstruktionsbedeutung die Komponente der Distanz beinhaltet ${ }^{3}$.

In (7) sehen wir wiederum Nachzustände, die durch das Perfekt - in seinen Resultatslesarten - wie auch durch passivische Zustandskonstruktionen realisiert werden können.

(7) Es ist spät, der Weißwein ist getrunken, die Geräusche sind verstummt, die Farben ergraut, die Leidenschaften verflogen. (Die Zeit 30.1.03: 41)

Hier liegt eine komplexe Zustandsbeschreibung vor, die aus einer adjektivischen KopulaPrädikativkonstruktion, einer passivischen Zustandskonstruktion und drei Perfektkonstruktionen besteht. Der Interpretationseffekt, die Zustände als simultan zu verstehen, entsteht, da nicht nur die adjektivische Kopula-Prädikativ- und die passivische Zustandskonstruktionen Zustände kodieren, sondern auch das Perfekt Zustände kodieren kann ${ }^{4}$.

In (8) hingegen entsteht ein anderer Bedeutungseffekt: Nach den Zustandsbeschreibungen der adjektivischen und passivischen Konstruktion folgen drei als sequentiell zu deutende Ereignisse, die durch das Präteritum mit seinen - wiederum perfektiven - Vergangenheitslesarten realisiert werden. Die sequentielle Interpretation entsteht, weil es sich bei verstummen, ergrauen und verfliegen um telische Verben handelt. Diese liefern lexikalische Grenzpunkte für die jeweiligen Teilsituationen, was dazu führt, dass deutsche L1-Sprecher diese präferiert als

\footnotetext{
${ }^{3}$ Vgl. genauer Abschnitt 3.

4 Vgl. genauer Abschnitt 3.
} 
einzelne abgeschlossene, ganze und damit nacheinander stattfindende Situationen perspektivieren.

(8) Es war spät, der Weißwein war getrunken, die Geräusche verstummten, die Farben ergrauten, die Leidenschaften verflogen.

In (9) schließlich haben wir ein typisches Beispiel für die Verwendung des Präteritums in literarischen Texten. Es handelt sich um den Beginn von Alessandro Bariccos Questa Storia in seiner deutschen Übersetzung.

(9) Lau die Mainacht Paris neunzehn hundert drei.

Hunderttausend Pariser ließen die Hälfte der Nacht in ihren Wohnungen zurück, als sie zu den Bahnhöfen Saint-Lazare und Montparnasse strömten. (Baricco 2008: 7)

Hier wird die dem Präteritum inhärente Distanz so genutzt, dass die Übersetzerin einen Perspektivwechsel von der faktischen Origo in die fiktive Welt des Romans, zur fiktiven Origo, vornimmt. Die Intuition, dass man sich perspektivisch sogleich in der fiktiven Welt des Textes befindet, entstünde mit Perfektkonstruktionen nicht.

\section{Zusammenhänge von Formen und Bedeutungen: Die Konstruktionsbedeutungen}

Die Gemeinsamkeiten und Unterschiede zwischen Perfekt und Präteritum lassen sich erfassen, indem man ihre Lesarten auf verschiedene Konstruktionsbedeutungen zurückführt. Unter einer Konstruktionsbedeutung wird hier nicht eine Invariante verstanden, die in jeder Lesart zu den gleichen Bedeutungseffekten führt (vgl. Welke 2010: 17), sondern eine prinzipiell zur Verfügung gestellte Bedeutung, die im jeweiligen Kontext unterschiedlich realisiert wird (vgl. Schumacher 2005: 142; 158/59). Die kontroverse Diskussion um Grundbedeutungen von Tempora soll hier nicht aufgegriffen werden (vgl. Ehrich 1992: 87-94 Thieroff 1992: 276280, Klein 2000: 360-365, Musan 2002: 21-26, Welke 2005: 32-56, 2010). Vielmehr erscheint es mir für Deutsch als Fremdsprache sinnvoll, Lehrenden als Hintergrundwissen das Bedeutungspotenzial von Tempora zu vermitteln, das durchaus systematisch auf morphologische Bestandteile zurückführbar ist. So wird Lehrenden eine Grundlage dafür an die Hand gegeben, Form-Bedeutungszusammenhänge im Sinne einer formfokussierten Steuerung salient $\mathrm{zu}$ machen und auf kognitive Lernerfragen zu reagieren, die auf dem Bemühen basieren, systematische Form-Bedeutungszusammenhänge in der L2 herzustellen (vgl. Eckerth 1998: 79, Handwerker 2002: 215, Schumacher 2005: 58-61).

Dieses Bedeutungspotenzial lässt sich an den Konstruktionsbedeutungen der Tempora ablesen. Zentrale Bestandteile sind hierbei die temporalen Relationen zwischen der Äußerungszeit ( $\mathrm{AZ}$ ), der Situationszeit (SZ) und der Tempuszeit (TZ) ${ }^{5}$ sowie die Komponente Nachzustand (RES). Die Tempuszeit ist die Zeit, die der Sprecher durch das finite Verb lokalisiert. Im Default-Fall ist es die Zeit, die er betrachtet und ins Zentrum seiner Äußerung stellt, d.h. eine Topic Time im Sinne Kleins (1994: 36-58, 2009: 46) ${ }^{6}$. Der Nachzustand ist das Resultat eines vorangegangenen Ereignisses, wie an den Beispielen (1) und (3) ersichtlich wurde. ${ }^{7}$

Beim Perfekt liegt die Situationszeit vor der mit dem Nachzustand verbundenen Tempuszeit, und die Tempuszeit liegt überlappend mit oder nach, - nicht vor - der Äußerungszeit: SZ vor TZ, RES \& TZ nicht vor ÄZ. Der Komplex aus Auxiliar und Partizip II-Morphem liefert den Bedeutungsbeitrag [Vorzeitigkeit in Bezug auf einen Nachzustand], das Präsensmorphem

\footnotetext{
${ }^{5}$ Vgl. Musan (2002: 3-8) für eine entsprechende Begrifflichkeit.

6 Die so verstandene dritte Bezugszeit bei den Tempora ermöglicht es, auch die Vergangenheitslesarten des Perfekts zu erklären, bei denen Adverbiale gerade nicht die Zeit spezifizieren, die das finite Verb lokalisiert, und somit nicht topikal sind (vgl. Schumacher 2005: 146-149 in Anlehnung an Musan 2002: 5, 84-87).

${ }^{7}$ Vgl. Klein (1994) und Musan (2002) für entsprechende theoretische Grundlagen und Schumacher (2005) für deren Anwendung in DaF.
} 
liefert den Beitrag [Nicht-Vergangenheit]. Dadurch werden einem Perfekt zwei Zeitintervalle zugeordnet: die Situationszeit selbst sowie die Zeit des Nachzustands dieser Situation. Je nach Kontext wird eins der beiden Zeitintervalle ins Zentrum der Äußerung gerückt und möglicherweise durch Adverbiale spezifiziert. Daraus entstehen dann Resultats- und Vergangenheitslesarten. In (1) und (3) macht der Sprecher eine Aussage über den Nachzustand, der in der Gegenwart (jetzt) bzw. in der Zukunft (gleich) liegt. Die resultative Gegenwartslesart lässt sich beispielsweise wie folgt veranschaulichen:

(1) Er hat sich damit jetzt als Politgangster entlarvt.

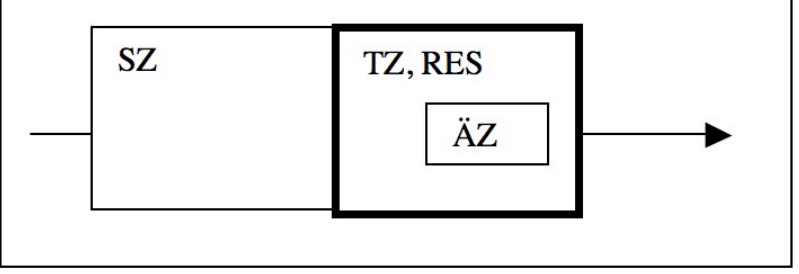

Abb. 3: Die resultative Gegenwartslesart des Perfekts

(5) ist eine Aussage über die vergangene Situation, die am Vortag (gestern) stattfand. Sie lässt sich in folgender Weise visualisieren:

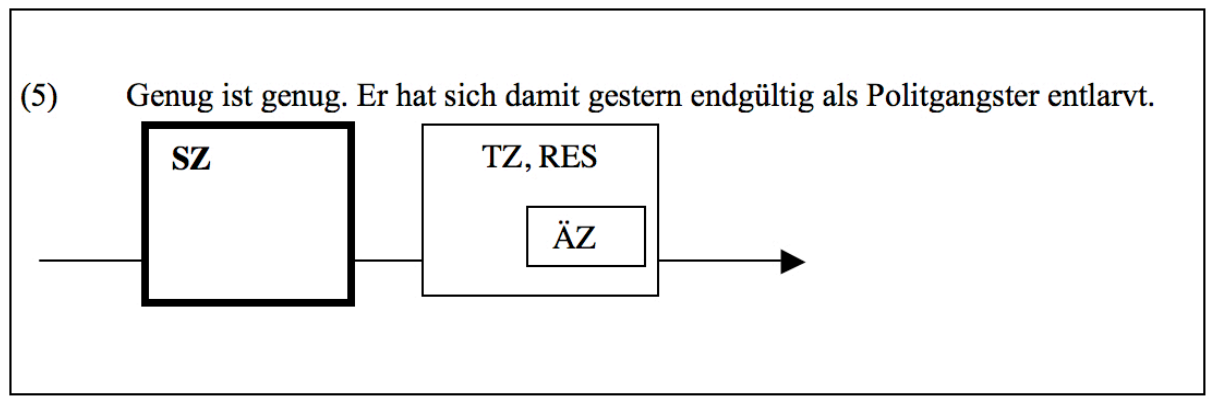

Abb. 4: Die (perfektive) Vergangenheitslesart des Perfekts

Bei Resultatslesarten spezifizieren Adverbiale die Tempuszeit, bei Vergangenheitslesarten die Situationszeit. Die jeweils spezifizierten Zeitintervalle sind in den Abbildungen 3 und 4 durch Fettdruck markiert.

Das Perfekt beinhaltet keinen aspektualen Wert in Bezug auf Perfektivität/Imperfektivität in seiner Konstruktionsbedeutung, d.h. es enthält keine Festlegung dahingehend, ob eine Situation mit oder ohne einen Endpunkt perspektiviert wird. ${ }^{8}$ Deshalb kann das Perfekt perfektive und imperfektive Vergangenheitslesarten realisieren. In (5) wird die Gesamtsituation des Sich-Entlarvens mit ihren beiden Zeitintervallen inkl. Endpunkt und Nachzustand ins Zentrum der Äußerung gerückt, so dass eine perfektive Vergangenheitslesart entsteht. Eine imperfektive Vergangenheitslesart liegt in (10) vor.

(10) Als wir nach Hause gekommen sind, hat Paolo gerade telefoniert.

Hier liefert der als-Satz die Tempuszeit für den Hauptsatz, d.h. er bezeichnet die Zeit, für die die Aussage im Hauptsatz Gültigkeit beansprucht. Perspektiviert wird so nur ein Moment der Gesamtsituation des Telefonierens, der Endpunkt wird ausgeblendet. Es handelt sich um eine Situation des Verlaufs oder ongoingness (vgl. Carroll/von Stutterheim 2003, Schmiedtová/von Stutterheim/Carroll 2011), die in vielen Sprachen mit einem grammatikalisierten

\footnotetext{
8 Vgl. Bertinetto (2001a, 2001b) für eine entsprechende theoretische Aspektdefinition und Schumacher (2005, 2008a, 2008b) für deren Anwendung in kontrastiven Studien Deutsch/Italienisch.
} 
Aspekt obligatorisch durch eine imperfektive oder progressive Form ausgedrückt würde. Eine entsprechende Lesart können auch Präteritalformen hervorrufen, was in (11) ersichtlich wird.

(11) Als wir nach Hause kamen, telefonierte Paolo gerade.

Das Präteritum enthält ebenso wenig wie das Perfekt eine aspektuale Festlegung in Bezug auf Perfektivität oder Imperfektivität in seiner Konstruktionsbedeutung. Folglich ermöglicht es perfektive und imperfektive Lesarten. Perfektive Präteritumlesarten liegen in (6) und (8) vor.

Die Konstruktionsbedeutung des Präteritums besteht darin, dass die Tempuszeit vor der ÄuBerungszeit liegt und die Situationszeit überlappend mit oder nach - nicht vor - der Tempuszeit: TZ vor $\ddot{A Z} \&$ SZ nicht vor TZ. Dies lässt sich an Abbildung 5 erkennen:

(6) Gestern passierte viel. Er entlarvte sich als Politgangster...

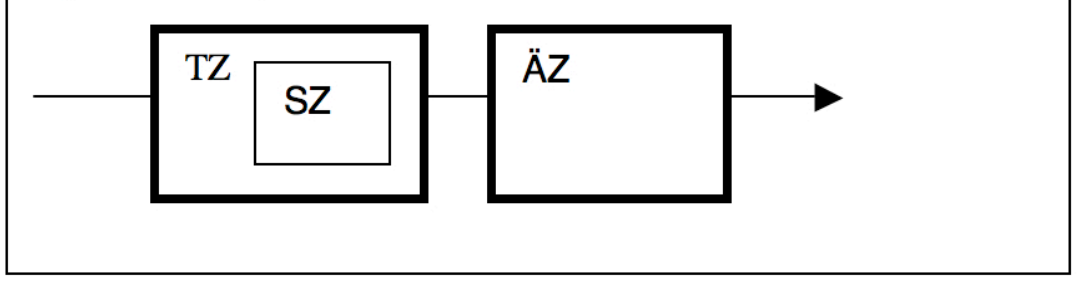

Abb. 5: Die (perfektive) Vergangenheitslesart des Präteritums

Das Präteritalmorphem markiert die Relation [Tempuszeit vor Äußerungszeit]. ${ }^{9}$ Diese - in Abbildung 5 durch Fettdruck hervorgehobene - Relation deute ich als Distanz, und Distanz hat eine temporale, eine relevanzbezogene und eine deiktische Funktion.

Die temporale Dimension besteht darin, dass die Tempuszeit immer mit einem zeitlichen Abstand vor der Äußerungszeit liegen muss. Dies ist - neben dem fehlenden Nachzustand in der Konstruktionsbedeutung - ein weiterer Grund dafür, dass (2) und (4) ungrammatisch sind.

Durch die relevanzbezogene Dimension von Distanz wird erfassbar, dass das Perfekt präferiert in dialogischen (oft mündlichen), das Präteritum hingegen bevorzugt in monologischen (oft: schriftlichen) Textsorten verwendet wird. ${ }^{10}$ Wie Wierzbicka/Schlegel (2008) ausführen, werden mit einem Präteritum Sachverhalte kodiert,

[...] zu denen der Sprecher innerlich Abstand gewonnen hat, bzw. die für die Gesprächsbeteiligten nicht weiter von Belang sind. (Wierzbicka/Schlegel 2008: 17)

Dies gilt vor allem für monologische Texte, die tendenziell eher in der Schriftsprache vorkommen. ${ }^{11}$ Beim Perfekt hingegen, das mündlich und schriftlich vorkommt, wird signalisiert, dass eine Äußerung für die Sprechsituation relevant ist, wie in (5) - vorstellbar in einem mündlichen Gespräch oder aber z.B. zu Beginn eines Zeitungsartikels - deutlich wurde. Mit einem Perfekt signalisiert der Schreiber eine subjektive Nähe zu seiner Äußerung, mit einem Präteritum eine subjektive Distanz, was an der Diskussion von (5) vs. (6) im Rahmen der Konzepte des Besprechens und Erzählens gezeigt wurde.

\footnotetext{
${ }^{9}$ Die Relation [Situationszeit nicht vor Tempuszeit] lässt sich mit Blick auf das ganze Tempussystem dadurch erklären, dass weder ein Auxiliar noch ein Partizip II vorhanden ist, um einen Nachzustand oder Vorzeitigkeit zu realisieren. Vgl. bereits Latzel (1977: 35f.) sowie Schumacher (2005: 187, 213).

10 Wie auch Willkop (2003a, b), Fandrych/Thurmair (2011: 258) und Attaviriyanupap (in diesem Band) ausführen, ist die verbreitete Zuordnung des Perfekts zur mündlichen und des Präteritums zur schriftlichen Sprache eine für Lernzwecke zu starke Reduktion.

11 Vgl. Wierzbicka/Schlegel (2008: 48-77) für eine korpusbasierte "Hitliste" von Verben, die in der gesprochenen Sprache präferiert im Perfekt bzw. Präteritum verwendet werden und für die Distanz keine Rolle spielt.
} 
Durch die deiktische Dimension der Distanz wird erklärbar, warum das Präteritum präferiert in der schriftlichen Sprache verwendet wird. Es ist ein anaphorisches Tempus, das nicht unmittelbar in Bezug auf das deiktische Zentrum einer Sprechsituation interpretiert werden kann, sondern auf eine von dieser Origo entfernt liegende Situation rückverweist. Ein spezifischer Effekt hierbei tritt häufig beim literarischen Erzählen hervor: Wie wir an (9) gesehen haben, eignet sich das Präteritum gut für die Verschiebung der Origo in die fiktive Welt eines literarischen Textes. Auch an (8) ist ein entsprechender Effekt zu erkennen.

Zusammenfassend möchte ich festhalten, dass sich Perfekt und Präteritum grammatisch hinsichtlich der nur vom Perfekt, nicht aber vom Präteritum zur Verfügung gestellten Bedeutungskomponente des Nachzustands unterscheiden. Im Gebrauch unterscheiden sie sich bzgl. des Faktors Distanz. Beide Dimensionen sind morphologisch verankert, was für Lernprozesse mit ihren Form-Bedeutungsverbindungen wichtig ist, und sollten in Deutsch als Fremdsprache als Teil des grammatischen und pragmatischen Wissens vermittelt werden. Bei sprachlich homogenen Lernergruppen - oder auch bei sprachlich heterogenen Lernergruppen, wenn Binnendifferenzierung umsetzbar ist - sollte in Abhängigkeit von den in der jeweiligen L1 grammatikalisierten Kategorien auch auf aspektuale Dimensionen bei den Vergangenheitslesarten fokussiert werden. Der folgende Abschnitt enthält erste Vorschläge dafür, Nachzustände, Distanz und Aspektualität in den Rahmen einer formfokussierten Steuerung von Perfekt und Präteritum zu integrieren.

\section{$4 \quad$ Formfokussierung beim Perfekt und Präteritum}

Unter einer formfokussierten Steuerung verstehe ich in Anlehnung an Ellis (2001)

$[\ldots]$ any planned or incidental instructional activity that is intended to induce language learners to pay attention to linguistic forms. (Ellis 2001: 1f.)

Bei aller Vielfalt an Positionen zu sprachlernfördernden didaktischen Strategien ist man sich einig darüber, dass Aufmerksamkeit (attention) und das Bemerken (noticing) eigener Wissenslücken (noticing the hole) oder einer Kluft zwischen der eigenen Lernersprache und der L2 (noticing the gap) Voraussetzungen dafür sind, dass eine Form-Bedeutungszuordnung überhaupt ins Arbeitsgedächtnis und sodann ins lernersprachliche System aufgenommen werden kann (vgl. Nassaji/Fotos 2007: 20, Handwerker/Madlener 2009: 26, Schormann/Schlak 2010: 16). Die verschiedenen didaktischen Strategien, die im Rahmen von Formfokussierung diskutiert und empirisch überprüft werden (vgl. für Überblicke Nassaji/Fotos 2007, Schormann/Schlak 2010) werden bei Ellis (2001: 16-26) drei verschiedenen Typen von Formfokussierung zugeordnet. Diese unterscheiden sich durch die beiden Kriterien "primärer Fokus" und "Verteilung der Aufmerksamkeit".

\begin{tabular}{|l|l|l|}
\hline Typ der Formfokussierung & Primärer Fokus & Verteilung der Aufmerksamkeit \\
\hline focus on forms & Form & intensiv \\
\hline Geplante Formfokussierung & Bedeutung & intensiv \\
\hline Spontane Formfokussierung & Bedeutung & extensiv \\
\hline
\end{tabular}

Abbildung 6: Typen von Formfokussierung (vgl. Ellis 2001: 17)

Der Typ focus on forms zeichnet sich dadurch aus, dass der primäre Fokus der Aufmerksamkeit von Lehrenden und Lernenden in einer bestimmten Unterrichtsphase auf der Formseite der Sprache liegt und dass einzelne, bereits im Vorfeld des Unterrichts ausgewählte sprachliche Strukturen intensiv im Klassenzimmer bearbeitet werden. Hierbei können explizitere oder implizitere didaktische Strategien angewandt werden, die den Input gezielt strukturieren oder den Output gezielt auslösen (vgl. Ellis 2001: 17-20, VanPatten 2004, Handwerker 2009: 97105). 
Auch bei der geplanten Formfokussierung werden vor dem Unterricht einzelne sprachliche Strukturen ausgewählt und dann im Klassenzimmer intensiv bearbeitet, jedoch geschieht dies innerhalb eines insgesamt bedeutungszentrierten Unterrichtsgeschehens. Inputflut und input enhancement sind vieldiskutierte didaktische Strategien, die darauf abzielen, den Input so zu modifizieren, dass im Rahmen von kommunikativen, auf die Bedeutung ausgerichteten Aufgaben Formmerkmale salient gemacht werden (vgl. Ellis 2001: 20-22, Wong 2005, Handwerker 2009: 98-99, Handwerker/Madlener 2009: 33-35). Auch outputorientierte Lernaufgaben (tasks) fokussieren eine spezifische L2-Struktur, die dafür notwenig ist, die insgesamt kommunikativ ausgerichtete Aufgabenstellung zu erfüllen (vgl. Eckerth 2003: 34-37, Ellis 2003: 152-157, Schoormann/Schlak 2010: 17).

Spontane Formfokussierung schließlich ist auch bedeutungszentriert, jedoch geschieht sie nicht geplant, sondern aus der Unterrichtssituation heraus. Da die Lehrenden auf Lerneräußerungen oder kommunikative Missverständnisse reagieren (reactive focus on form) oder aber mögliche Lernschwierigkeiten bei Form-Bedeutungszuordnungen in einer entstandenen Interaktion vorhersehen und "präventiv" fokussieren (pre-emptive focus on form), wird die Aufmerksamkeit von Lernenden auf eine Vielzahl an sprachlichen Strukturen gelenkt (vgl. Ellis 2001: 22-24, Nassaji/Fotos 2007: 13). In der Diskussion um spontane Formfokussierung werden verschiedene Feedbacktypen im Klassenzimmer empirisch untersucht. Eine besondere Rolle spielen hier recasts ${ }^{12}$ einerseits und die Frage negativer Evidenz andererseits (vgl. Doughty/Varela 1998, Ellis 2001: 22-25, Ellis 2008: 226-232, Schoormann/Schlak 2010: 1719).

Um die Bedeutungs- und Gebrauchskomponenten Nachzustand, Distanz und Aspektualität in einer formfokussierten Steuerung von Perfekt und Präteritum zu vermitteln, bieten sich - was die Sprachrezeption betrifft - beispielsweise die Verfahren der Inputflut und des input enhancement an. Um die Aufmerksamkeit von Lernenden auf die Partizipien und Auxiliare zu lenken, die gegenwärtige und zukünftige Resultatslesarten ermöglichen, sowie auf telische Verben und Gegenwarts- bzw. Zukunftsadverbiale, die typischerweise zu Resultatslesarten führen, eignet sich eine reine Inputflut, bei der Lernenden viele Sätze wie (1) oder (3) oder aber Texte präsentiert werden, in denen das Perfekt im Zusammenhang mit den genannten Verbtypen und Adverbialen gehäuft vorkommt. Ein solches Verfahren der geplanten Formfokussierung ist vergleichsweise unaufdringlich. Die Verfahren der Formfokussierung innerhalb eines insgesamt bedeutungszentrierten Unterrichtsgeschehens lassen sich nach ihrem Grad an Aufdringlichkeit, mit der in das kommunikative Geschehen eingegriffen wird, klassifizieren (vgl. Doughty/Williams 1998: 258, Handwerker 2009: 98, Handwerker/Madlener 2009: 33, Schifko 2011: 145). Ein etwas aufdringlicheres Verfahren ist das input enhancement, bei dem - in unserem konkreten Fall - die Auxiliare, Partizipien und Adverbiale graphisch (oder intonatorisch) markiert werden könnten, um die Aufmerksamkeit von Lernenden expliziter auf die Formen zu lenken, die für deutsche L1-Sprecher präferiert zu Resultatslesarten führen. Um es Lernenden auch zu ermöglichen, zu bemerken, dass sowohl das Perfekt als auch das sog. Zustandspassiv Nachzustände von vorangegangenen Situationen ausdrücken und dass dies zudem oftmals der gleichen Form entspricht (sein + Partizip II), bietet es sich an, Lernende mit entsprechendem Input (vgl. (8)) zu fluten und/oder ihn zudem graphisch zu markieren.

Entsprechende Form-Bedeutungszusammenhänge lassen sich auch im Rahmen von focus on forms salient machen. In Eckerths (1998) Beitrag zu sprachbezogenen Lernerfragen findet sich im Rahmen einer Unterrichtseinheit zum sein-Perfekt der Verweis einer Lernerin auf das Zustandspassiv:

\footnotetext{
${ }^{12}$ Bei recasts ("Umgestaltungen") formulieren Lehrende eine fehlerhafte Lerneräußerung um und korrigieren sie dabei implizit, indem sie einzelne Veränderungen vornehmen, ohne dabei die zentrale Bedeutung der ursprünglichen Äußerung zu modifizieren.
} 
L: so dann ergänzen wir noch diese Liste (deutet auf die Tafel) also ich habe bei eins geschrieben Verben die Ortsveränderung bedeuten aber es gibt noch eine andere Gruppe von Verben die auch das Perfekt mit sein bilden

S2: etwas das ist schon passiert das Fenster ist geöffnet

L: $\quad$ mhm das ist extra das ist ein extra Problem (Eckerth 1998: 79)

Lernerin und Lehrerin sind sich in dieser Unterrichtsphase darüber bewusst, dass die Formseite der Sprache im Fokus liegt. Es ist davon auszugehen, dass durch die Formfokussierung bei der Lernerin eine Aufmerksamkeit für Form-Bedeutungszusammenhänge vorliegt. Dies zeigt sich durch die ihrer Äußerung zugrunde liegende Hypothese, nach der die an der morphosyntaktischen Oberfläche gleichen Konstruktionen (sein-Perfekt und Zustandspassiv) die gleiche Bedeutung haben (vgl. Schumacher 2005: 59). In dieser Interaktion hätte die Lehrerin durch eine weitere Formfokussierung - etwa in Form einer metasprachlichen Erklärung - ein noticing dafür hervorrufen können, dass sowohl das Perfekt als auch das Zustandspassiv Nachzustände von vorangegangenen Ereignissen kodieren können.

Was das Präteritalmorphem angeht, das Distanz signalisiert, so bieten sich wiederum die Verfahren der Inputflut und des input enhancement an. Gegenstand könnten erzählende kurze Sequenzen wie (6) oder aber literarische Abschnitte wie (9) sein, um die Aufmerksamkeit von Lernenden vergleichsweise unaufdringlich darauf zu lenken, dass das Präteritum subjektive Distanz im Sinne von Nicht-Relevanz bzw. für eine Verschiebung der Origo in eine fiktive Welt ermöglicht. Um für Lernende weitere Verbindungen von Formen und Bedeutungen salient zu machen, eignen sich auch Textanfänge mit Plusquamperfektkonstruktionen, die - wie auch das Präteritum, das ja Teil ihrer Konstruktion ist - Distanz signalisieren (vgl. Schumacher 2005: 208-210).

Eine aufdringlichere Form der geplanten Formfokussierung ist das Dictogloss (vgl. Doughty/Williams 1998b: 258). Bei dieser didaktischen Strategie wird Lernenden ein Text diktiert, der dann gemeinsam mit einem anderen Lerner rekonstruiert werden soll. Wie Swain (1998) zeigt, führt diese Aufgabenstellung zu einem kooperativen Arbeiten zwischen Lernenden, bei dem die Aufmerksamkeit im Rahmen einer bedeutungszentrierten Aufgabe immer wieder auf die Formseite gerichtet ist und die Lernenden in dem Moment metasprachliche Gegenstände aushandeln, in dem sie eine Lücke in ihrer Lernersprache entdecken (vgl. Swain 1998: 6667). Bei dieser Art der geplanten Formfokussierung bietet es sich für den Lerngegenstand Präteritum an, einen Text zu diktieren und rekonstruieren zu lassen, der erzählende Elemente enthält sowie solche, die für die jeweiligen Sprechzeiten im Diskurs (vgl. Wierzbic$\mathrm{ka} /$ Schlegel 2008) relevant sind. So können Lernende dazu gebracht werden, innerhalb ihres bedeutungszentrierten Dialogs immer wieder ihre Aufmerksamkeit auf die morphologische Realisierung von Distanz im Präteritum zu lenken und ihr metasprachliches Wissen jeweils dann zu aktivieren, wenn sie eine Lücke in ihrer Lernersprache bemerkt haben.

Formfokussierung zu aspektualen Bedeutungskomponenten von Perfekt und Präteritum schließlich sollte idealerweise unter Berücksichtung der L1 der Lernenden erfolgen. So kann die sprachspezifische Prägung berücksichtigt werden, die die Aufmerksamkeit von Lernenden steuert. In verschiedenen Studien zur sprachspezifischen Perspektivierung hat sich gezeigt, dass der grammatische Aspekt in der L1 Lernende auch in der L2 beeinflusst (vgl. Carroll/von Stutterheim 2003, von Stutterheim/Carroll 2006, 2007, Reznicek 2008). Bei italienischen Lernenden beispielsweise sollten im Rahmen von Inputfluten und input enhancement lexikalische Ausdrucksmittel für imperfektive Lesarten des Perfekts sowie perfektive Lesarten des Präteritums salient gemacht werden. Denn das italienische Passato Prossimo ist im Gegensatz zum deutschen Perfekt eine perfektive Tempuskonstruktion, die nur Lesarten mit Abgeschlossenheits- und Resultativitätseffekten, jedoch keine imperfektiven Lesarten zulässt, und das italienische Imperfetto ermöglicht im Gegensatz zum deutschen Präteritum keine perfektiven 
Lesarten (vgl. Schumacher 2008b). In meiner Studie zur Rezeption des deutschen Präteritums durch italienische Lernende ist deutlich geworden, dass Sätze im Präteritum mit Adverbialen oder Verben, die Endpunkte bezeichnen, durch italienische L1-Sprecher eher habituell interpretiert werden, durch deutsche L1-Sprecher hingegen eher abgeschlossen (vgl. Schumacher 2008a).

(12) Franza tanzte bis Mitternacht Tango.

Wegen des Kontrastmangels zwischen dem Präteritum und dem Imperfetto tendieren italienische Deutschlernende dazu, die Gebrauchsbeschränkungen des Imperfetto auf die des Präteritums zu übertragen: Da Franza ballava il tango fino a mezzanotte nur habituell interpretiert werden kann, wird auch ein Satz wie (12) von italienischen Lernenden häufig habituell interpretiert. Deutsche L1-Sprecher hingegen tendieren dazu, (12) als Äußerung über ein einmalige Ereignis - beispielsweise vom Vortag - zu verstehen.

Um die nativen Gebrauchspräferenzen des Präteritums für italienische Lernende salient zu machen, bietet es sich an, Lernende mit Sätzen oder Texten, die perfektive Vergangenheitslesarten des Präteritums enthalten, zu fluten und darüber hinaus Grenzadverbiale wie bis Mitternacht und telische Verben graphisch hervorzuheben. Auch die lexikalischen Mittel, die zu imperfektiven Lesarten des Perfekts führen (Adverbiale wie gerade, zu der Zeit, temporale als-Sätze mit atelischen Verben, Verlaufskonstruktionen wie $a m+$ Infinitiv + sein, vgl. Schumacher 2005: 276) könnten in hoher Frequenz im Input geliefert und zusätzlich markiert werden.

\section{$5 \quad$ Ausblick}

Die hier skizzierten ersten Ideen zu einer Formfokussierung der Komponenten Nachzustand, Distanz und Aspektualität beim Perfekt und Präteritum harren natürlich noch einer Ergänzung in verschiedener Hinsicht: Sie harren der Entwicklung konkreter formfokussierter Aufgaben, der empirischen Überprüfung ihres Sprachförderpotenzials und ihrer didaktischen Umsetzung. Studierende in der Lehrerausbildung sollten sowohl für die linguistischen Grundlagen von Perfekt und Präteritum als auch für die verschiedenen Möglichkeiten, Formfokussierung im Klassenzimmer umzusetzen, sensibilisiert werden. Hierbei sollte auch ein Bewusstsein für Feedbackoptionen im Rahmen einer spontanen Formfokussierung geschaffen werden; wie beispielsweise Doughty/Varela (1998) zeigen, sind recasts gerade bei komplexen Lerngegenständen wie Tempora lernfördernd. Entsprechende Beiträge aus der Forschung zum gesteuerten Zweitspracherwerb und aus der Didaktik würden dazu beitragen, die Diskrepanz zwischen der Darstellung von Perfekt und Präteritum in Lehrmaterialien, in der Linguistik und in der Zweitspracherwerbsforschung zu überwinden und so eine lerntheoretisch fundierte Steuerung dieses nach wie vor faszinierenden Lerngegenstands zu ermöglichen. ${ }^{13}$

\footnotetext{
${ }^{13}$ Ich danke Michael Schümann, Klaus Peter, Gabriela Perrig und einem anonymen Gutachter/einer anonymen Gutachterin herzlich für äußerst hilfreiche Hinweise zu meinen Ausführungen.
} 


\section{Literatur}

Bardovi-Harlig, Kathleen (2000): Tense and Aspect in Second Language Acquisition: Form, Meaning, and Use. Oxford: Blackwell.

Baricco, Alessandro (2008): Diese Geschichte. Aus dem Italienischen von Annette Kopetzki. München: Carl Hanser Verlag.

Bertinetto, Pier Marco (2001a): "Il verbo". In: Renzi, Lorenzo/Salvi, Giampaolo/Cardinaletti, Anni (eds.): Grande grammatica italiana di consultazione. Nuova edizione. Vol. II. I sintagmi verbale, aggettivale, avverbiale. La subordinazione. Bologna, Il Mulino: 13-161.

Bertinetto, Pier Marco (2001b): "On a frequent misunderstanding in the temporal-aspectual domain: The 'perfective-telic confusion'". In: Ceccetto, Carlo/Chierchia, Bennaro/Guasti, Maria Teresa (eds.): Semantic Interfaces. Reference, Anaphora and Aspect. Standford, CSLI Publications: $177-210$.

Bertinetto, Pier Marco/Delfitto, Denis (2000): "Aspect vs. Actionality: Why they should be kept apart". In Dahl, Östen (ed.): Tense and Aspect in the Languages of Europe. Berlin, de Gruyter: 189-226.

Carroll, Mary/Stutterheim, Christiane von (2003): "Typology and information organisation: perspective taking and language-specific effects in the construal of events". In: Giacalone Ramat, Anna (ed.): Typology and Second Language Acquisition. Berlin, Mouton de Gruyter: $365-402$.

Doughty, Catherine/Varela, Elizabeth (1998): "Communicative focus on form". In: Doughty/Williams (eds.): 114-138.

Doughty, Catherine/Williams, Jessica (eds.) (1998a): Focus on Form in Classroom Second Language Acquisition. Cambridge: Cambridge Univ. Press.

Doughty, Catherine/Williams, Jessica (1998b): "Pedagogical choices in focus on form". In: Doughty/Williams (eds.): 197-262.

Eckerth, Johannes (1998): Kognitive Aspekte sprachbezogener Lernerfragen. Interaktion und Kognitivon im Deutsch-als-Fremdsprache-Unterricht. Baltmannsweiler: Schneider Verlag Hoehengehren.

Eckerth, Johannes (2003): Fremdsprachenerwerb in aufgabenbasierten Interaktionen. Tübingen: Narr.

Ehrich, Veronika (1992): Hier und Jetzt. Studien zur lokalen und temporalen Deixis im Deutschen. Tübingen: Niemeyer.

Ellis, Rod (ed.) (2001): Form-focused instruction and Second Language Learning. Oxford: Blackwell Publishing.

Ellis, Rod (2003): Task-based Language Learning and Teaching. Oxford: Oxford University Press.

Ellis, Rod (2008): The Study of Second Language Acquisition. Oxford: Oxford University Press.

Fandrych, Christian/Thurmair, Maria (2011): Textsorten im Deutschen. Linguistische Analysen aus sprachdidaktischer Sicht. Tübingen: Stauffenburg.

Fotos, Sandra/Nassaji, Hossein (eds.) (2007): Form-Focused Instruction and Teacher Education. Studies in Honor of Rod Ellis. Oxford: Oxford Univ. Press.

Handwerker, Brigitte (2002): "Chunks, Raster und Regeln: Vom Lexikon zur Grammatik in der Fremdsprachenvermittlung". In: Börner, Wolfgang/Vogel, Klaus (eds.): Grammatik und Fremdsprachenerwerb. Tübingen, Narr: 207-230.

Handwerker, Brigitte (2003): "Telizität im Deutschen - ein Lerngegenstand". Deutsch als Fremdsprache 40.3: 141-147.

Handwerker, Brigitte (2009): "Sprachunterricht als Instruktion zur Inputverarbeitung". Zeitschrift für Linguistik und Literaturwissenschaft 39.153: 96-111. 
Handwerker, Brigitte/Madlener, Karin (2009): Chunks für DaF. Theoretischer Hintergrund und Prototyp einer multimedialen Lernumgebung (inclusive DVD). Baltmannsweiler: Schneider Verlag Hohengehren.

Klein, Wolfgang (1994): Time in Language. London/New York: Routledge.

Klein, Wolfgang (2000): "An analysis of the German Perfekt". Language 76: 358-382.

Klein, Wolfgang (2009): "How time is encoded". In: Klein, Wolfgang/Li, Ping (eds.): The Expression of Time. Berlin/New York, Mouton de Gruyter: 39-82.

Latzel, Sigbert (1977): Die deutschen Tempora Perfekt und Präteritum. Eine Darstellung mit Bezug auf Erfordernisse des Faches Deutsch als Fremdsprache. Ismaning: Max Hueber Verlag.

Musan, Renate (1999): "Die Lesarten des Perfekts". Zeitschrift für Literaturwissenschaft und Linguistik 29.113: 6-51.

Musan, Renate (2002): The German Perfect. Its Semantic Composition and its Interactions with Temporal Adverbials. Dordrecht: Kluwer Academic Publishers.

Nassaji, Hossein/Fotos, Sandra (2007): "Issues in form-focused instruction and teacher education". In: Fotos / Nassaji (ed.), 7-16.

Reznicek, Marc (2008): Thinking L1 for Speaking L2. Der Einfluss des muttersprachlichen Aspektsystems bei spanischsprachigen Deutschlernern. Unveröffentlichte Magisterarbeit Humboldt-Universität zu Berlin.

Schifko, Manfred (2011): Formfokussierung als fremdsprachendidaktisches Konzept. Psycholinguistische Modellierung und Taxonomie von Unterrichtstechniken. Hamburg: Verlag Dr. Kovac.

Schmiedtová, Barbara/von Stutterheim, Christiane/Carroll, Mary (2011): "Language-specific patterns in event construal of advanced second language speakers". In: Pavlenko, Aneta (ed.): Thinking and Speaking in Two Languages. Bristol u.a.: Multilingual Matters, 66107.

Schoormann, Mathias/Schlak, Torsten (2010): "Weiterentwicklung der Lernersprache auf Basis der counter-balanced instruction - zum Spannungsverhältnis von formen- und inhaltsfokussierten Unterrichtsformen im Zweit- und Fremdsprachenunterricht". Deutsch als Zweitsprache 1/2010: 15-22.

Schumacher, Nicole (2005): Tempus als Lerngegenstand. Ein Modell für Deutsch als Fremdsprache und seine Anwendung für italienische Lernende. Tübingen: Gunter Narr Verlag.

Schumacher, Nicole (2008a): "The role of grammatical aspect in the comprehension of tense: Evidence from advanced learner varieties L1 Italian - L2 German". In: Walter, Maik/Grommes, Patrick (eds.): Fortgeschrittene Lernervarietäten. Korpuslinguistik und Zweitspracherwerbsforschung. Tübingen, Niemeyer: 73-92.

Schumacher, Nicole (2008b): "Vergangenheitstempora im Italienischen und Deutschen". In: Nied Curcio, Martina (ed.): Ausgewählte Phänomene zur kontrastiven Linguistik Italienisch-Deutsch. Ein Studien- und Übungsbuch für italienische DaF-Studierende. Mailand, FrancoAngeli: 81-97.

Schumacher, Nicole (2009): "Perspektiven für Studien zum Gebrauch der deutschen Vergangenheitstempora durch russische L1-Sprecher". In: Goncharova, Eugenia/Schumacher, Nicole/Strietz, Monika et al. (eds.): Arbeitspapiere GIP Herzen - Humboldt. Themenheft 1: Tempus - Metapher - Text. St. Petersbug, Izdate'stvo SPbGPU: 28-46.

Schwarze, Christoph (1995): Grammatik der italienischen Sprache. 2., verbesserte Auflage. Tübingen: Niemeyer.

Sorace, Antonella (2003): "Near-nativeness". In: Doughty, Catherine (ed.): The Handbook of Second Language Acquisition. Oxford, Blackwell: 130-151.

Squartini, Mario (1995): "Tense and aspect in Italian". In: Thieroff, Rolf (ed.): Tense Systems in European Languages II. Tübingen, Niemeyer: 117-134. 
Starren, Marianne (2001): The Second Time. The Acquisition of Temporality in Dutch and French as a Second Language. Utrecht: LOT.

Stutterheim, Christiane von/Carroll, Mary (2006): "The impact of grammaticalised temporal categories on ultimate attainment in advanced L2-acquisition". In: Byrnes, Heidi (ed.): Educating for advanced foreign language capacities: constructs, curriculum, instruction, assessment. Georgetown: University Press: 40-53.

Stutterheim Christiane von/Carroll, Mary (2007): "Durch die Grammatik fokussiert". Zeitschrift für Literaturwissenschaft und Linguistik 36.145: 35-60.

Stutterheim, Christiane von/Carroll, Mary/Klein, Wolfgang (2009): "New perspectives in analyzing aspectual distinctions across languages". In: Klein, Wolfgang/Li, Ping (eds.): The Expression of Time. Berlin/New York, Mouton de Gruyter: 195-215.

Swain, Merrill (1998): Focus on form through conscious reflection. In: Doughty/Williams (eds.), 64-82.

Thieroff, Rolf (1992): Das finite Verb im Deutschen. Tempus - Modus - Distanz. Tübingen: Narr.

VanPatten, Bill (ed.) (2004): Processing Instruction. Theory, Research, and Commentary. Lawrence Erlbau, Mahwahl, N.J.

Weinrich, Harald (1993): Textgrammatik der deutschen Sprache. Mannheim: Dudenverlag.

Welke, Klaus (2005): Tempus im Deutschen. Rekonstruktion eines semantischen Systems. Berlin: Walter de Gruyter.

Welke, Klaus (2010): "Contra Invarianz - Tempus im DaF (II): Perfekt und Präteritum". Deutsch als Fremdsprache 47.1: 17-25.

Wierzbicka, Mariola/Schlegel, Dorothee (2008): Sprechzeiten im Diskurs. Zum absoluten und relativen Gebrauch der Tempora in der gesprochenen deutschen Sprache. München: Iudicium.

Williams, Jessica (2008): "Form-focused instruction". In: Hinkel, Eli (ed.): Handbook of Research in Second Language Teaching and Learning. New York, Routledge: 671-692.

Willkop, Eva-Maria (2003a): "Perfekte Geschichten - Tempuswahl in Erzähltexten". In: Thurmair, Maria/Willkop, Eva-Maria (eds.): Am Anfang war der Text. 10 Jahre "Textgrammatik der deutschen Sprache". München, Iudicium: 235-258.

Willkop, Eva Maria (2003b): "Anwendungsorientierte Textlinguistik - Am Beispiel von Textsorten, Isotopien, Tempora und Referenzformen". GFL 2: 84-11.

Wong, Winnie (2005): Input Enhancement. From Theory and Research to the Classroom. New York: McGraw-Hill. 